\title{
Evaluation of the Adding Paracetamol to Dexmedetomidine in Pain Management After Adult Cardiac Surgery
}

\author{
Fatemehshima Hadipourzadeh ${ }^{1, *}$, SeyedMehdi Mousavi ${ }^{1}$, Avaz Heydarpur ${ }^{1}$, Ali Sadeghi ${ }^{1}$ and Rasool \\ Ferasat-Kish ${ }^{1,2}$ \\ ${ }^{1}$ Cardiac Anesthesia Department, Rajaie Cardiovascular Medical and Research Center, Iran University of Medical Sciences, Tehran, Iran \\ ${ }^{2}$ Pain Research Center, Department of Anesthesiology and Pain Medicine, Iran University of Medical Sciences, Tehran, Iran \\ "Corresponding author: Cardiac Anesthesia Department, Rajaie Cardiovascular Medical and Research Center, Iran University of Medical Sciences, Vali-Asr Street, Tehran, Iran. \\ Tel: +98-2123923740, Fax:+98-21 22663293, Email: shimahadipoorzadeh@gmail.com
}

Received 2020 November 15; Revised 2021 May 29; Accepted 2021 May 30.

\begin{abstract}
Background: Postoperative pain control after cardiac surgery is usually based on Opioids. These drugs are associated with side effects, and the use of drugs with fewer side effects is important for analgesia. Dexmedetomidine and paracetamol have fewer side effects than opioids.

Objectives: The aim of the study was to evaluate the adding paracetamol to dexmedetomidine continuous infusion pump for pain management after adult cardiac surgery.

Methods: In this study, 100 patients were divided into two groups. One group received a continuous infusion of dexmedetomidine and paracetamol (DP), and the other received dexmedetomidine(D). These two groups were evaluated for MAP, HR, and the need for prescribing opioids before and after extubation. Also, duration of intubation and pain before extubation and 36 hours after every 4 hours.

Results: Patients in the DP group had lower mean MAP and HR during intubation period than the D group and needed fewer opioids and doses of opioids in addition to drug study infusion pre- $(\mathrm{P}=0.001)$ and post-extubation $(\mathrm{P}=0.001$ and $\mathrm{P}=0.022$, respectively). The DP group patients were extubated earlier $(\mathrm{P}=0.001)$. After extubation, the DP group had less pain than the $\mathrm{D}$ group.

Conclusions: This study showed that adding paracetamol to the dexmedetomidine infusion pump can provide better analgesia.
\end{abstract}

Keywords: Dexmedetomidine, Paracetamol, Cardiac Surgery, Pain Management

\section{Background}

Since plenty of cardiac surgeries are performed annually, and a relationship exists between proper control of postoperative pain and improvement in clinical outcomes, anesthesiologists need to make progress in learning a variety of anesthesia and pain control techniques (1). Several factors contribute to postoperative pain, including sternotomy, retraction of sternum and rib, pericardiotomy, harvest of internal mammary artery, harvest of saphenous vein, manipulation during surgery on parietal pleura, insert chest tube, and other musculoskeletal injuries during surgery. Gold standard for pain control after cardiac surgery is usually based on opioids that are associated with side effects such as constipation, nausea and vomiting, dizziness and mental confusion, and respiratory depression, which can affect the patient's initial recovery and discharge time $(2,3)$. Poor treatment of acute pain affects both the patient's health and the progression of acute pain to chronic pain $(4,5)$. Injectable NSAIDs, such as ketorolac, can cause gastrointestinal ulcers, renal failure, and platelet dysfunction. Therefore, it is important to know the drugs that do not cause these problems. Dexmedetomidine has been proven that improves analgesia and anesthesia and reduce the need for opioids $(6,7)$. It can inhibit nausea and vomiting, and shivering after surgery. It also reduces postoperative delirium. This drug, when is used alone, can cause analgesia and sedation without respiratory depression. Paracetamol is a drug with analgesic and antipyretic effects, with no anti-inflammatory effects (8) It is a logical analgesic for mild to moderate pain after surgery, but in severe pain should be used with other drugs $(9,10)$. In this study, we aimed to evaluate the effect of these drugs on analgesia after cardiac surgery.

\section{Objectives}

The aim of the study was to evaluate the adding paracetamol to dexmedetomidine in pain management after adult cardiac surgery. 


\section{Methods}

This study was approved by the Ethics Committee and was performed from May 2019 to July 2019. Inclusion criteria were patients undergoing elective cardiac surgery, coronary artery bypass graft (CABG) surgery, cardiac valve surgery, $C A B G$, and cardiac valve surgery at the same time. Exclusion criteria were age less than 18 years and age over 80 years, $\mathrm{Cr}>2 \mathrm{mg} / \mathrm{dL}$, Ejection fraction $<40 \%$, plasma bilirubin $>1.8 \mathrm{mg} / \mathrm{dL}$, liver enzymes (AST and ALT) $>1.5$ of the upper limit of normal, coagulation disorders, severe respiratory diseases, history of cerebrovascular accident and transient ischemic attack about six months ago, emergency cardiac surgery, aneurysm, and dissection surgery, congenital cardiac surgery, need to reoperation due to postoperative bleeding, drug abuse, and patient refusal. Both groups had similar anesthesia protocols. In the D group, continuous infusion pump contains dexmedetomidine 400 mcg dexmedetomidine (Precedex®, Hospira, Illinois, USA), and in the DP group, contained dexmedetomidine $400 \mathrm{mcg}$ and paracetamol (Paracetamol Zolben, Switzerland) 2 gr, at $100 \mathrm{~mL}$ normal saline, and which were infused at a rate of $0.5-1 \mathrm{mcg} / \mathrm{kg} / \mathrm{h}$ for 36 hours from the time of arrival to the ICU. Mean arterial pressure and heart rate (MAP, HR) were evaluated under mechanical ventilation and were evaluated every two hours from the time of admission to the ICU until the time of extubation. In cases of need for more sedation, propofol (35 mcg/ $/ \mathrm{kg} / \mathrm{min}$ ) was infused, and morphine i.v. (0.05 - $0.1 \mathrm{mg} / \mathrm{kg}$ ) was administered to those who need more analgesia. Patients who needed propofol and morphine were evaluated in both groups. The duration of intubation, the intensity of pain score, and the need for more opioids after tracheal extubation were evaluated every 4 hours for 36 hours. Numerical Rating Scale (NRS) was used to evaluate pain after extubation ( 0 - 1 no pain, 2 - 3 mild pain, 4 - 5 mild to moderate pain, 6 - 7 moderate pain, 8 - 9 moderate to severe pain, 10 severe pain).

\section{Results}

Demographic data were not significant between the two groups (Table 1). Mean arterial pressure in the DP group was less than the D group, and a significant difference was seen (Table 2), but there was no significant difference for heart rate (Table 2). The need for propofol during the intubation period was not significant $(\mathrm{P}=0.4)$. The need for morphine during intubation was $84 \%$ in the D group and 50\% in the DP group, which was a significant difference $(P=0.001)$. Dose of morphine received in the $D$ group was significantly higher than in the DP group $(\mathrm{P}=$ $0.001)$. The number of patients with mild pain $(2-3)$, and mild to moderate ( 4 - 5) pain score in the DP group was significantly more than the $\mathrm{D}$ group (Table 3 ). Based on the need for morphine after extubation, it was shown that in the D group was $96 \%$ and in the DP was $58 \%$ that was a significant difference $(\mathrm{P}=0.001)$. Dose of morphine after extubation in the D group was more than the DP group, and this relationship was significant $(\mathrm{P}=0.022)$. Also, the duration of intubation in the DP was shorter than in the D group, and this difference was significant between the two groups $(\mathrm{P}=0.001)$.

\begin{tabular}{lccc}
\hline \multicolumn{4}{l}{ Table 1. Demographic Parameters in Two Study Groups ${ }^{\mathrm{a}}$} \\
\hline DP Group $(\mathbf{N}=\mathbf{5 0})$ & D Group $(\mathbf{N}=\mathbf{5 0})$ & P-Value \\
\hline Sex M:F & $31: 19$ & $30: 20$ & 0.50 \\
Age (y) & $53.8 \pm 9.7$ & $57.2 \pm 10.2$ & $0.05^{\mathrm{b}}$ \\
BMI & $25.5 \pm 4.1$ & $27.1 \pm 3.5$ & 0.06 \\
\hline
\end{tabular}

${ }^{\mathrm{a}}$ Data are presented as No. or mean $\pm \mathrm{SD}$.

${ }^{\mathrm{b}}$ Significant P-value

\section{Discussion}

This study shows that adding paracetamol to the dexmedetomidine in a continuous infusion pump can provide better analgesia, reduce the need for opioids, and reduce the duration of intubation. Improperly controlled pain is associated with activation of the sympathetic nervous system and increases the hormonal response to stress. This response may be associated with several postoperative complications, including myocardial ischemia, cardiac arrhythmias, coagulation disorder, pulmonary problems, and delirium, and infection of the operation site $(11,12)$. Severe pain also reduces patient satisfaction, delays the onset of movement, and acute pain continues as chronic pain $(10,13)$. Pain can cause atelectasis, pulmonary infection, and reduced ability to produce enough sputum. Lack of movement due to pain can cause deep venous thrombosis, which can be associated with pulmonary thrombosis. Pain can also cause myocardial infarction, insomnia, and behavioral disorders (14). This study showed the group receiving continuous infusion with a mixture of dexmedetomidine and paracetamol had more stable hemodynamics during intubation than the dexmedetomidine alone. Furthermore, a combination mixture group needed fewer opioids and became extubated sooner, and after extubation they had less pain and need fewer opioids.

Dexmedetomidine is known as a selective alpha-2 agonist, which prevents the release of norepinephrine in the central nervous system, improves analgesia and anesthesia, and reduces opioid requirement (15). It can inhibit nausea and vomiting, and shivering after surgery. It also reduces postoperative delirium (16). Several studies have shown that this drug, when is used alone, can cause analgesia and sedation without respiratory depression $(17,18)$. 


\begin{tabular}{|c|c|c|c|}
\hline MAP and HR & DP Group $($ Mean \pm SD $)$ & D Group $($ Mean $\pm S D)$ & P-Value \\
\hline \multicolumn{4}{|l|}{ MAP (bpm) } \\
\hline $2 \mathrm{~h}$ & $81.3 \pm 9.8$ & $94.7 \pm 11.6$ & $0.001^{\mathrm{a}}$ \\
\hline $4 \mathrm{~h}$ & $82.7 \pm 11.4$ & $93.8 \pm 12.1$ & $0.001^{\mathrm{a}}$ \\
\hline $6 \mathrm{~h}$ & $81.4 \pm 10.2$ & $95.5 \pm 9.3$ & $0.001^{\mathrm{a}}$ \\
\hline $8 \mathrm{~h}$ & $79.4 \pm 9.3$ & $97.3 \pm 11.1$ & $0.001^{\mathrm{a}}$ \\
\hline $10 \mathrm{~h}$ & $80.6 \pm 12.1$ & $104.7 \pm 5.5$ & $0.030^{\mathrm{a}}$ \\
\hline $12 \mathrm{~h}$ & $87.0 \pm 12.1$ & $97.0 \pm 11.3$ & $0.050^{\mathrm{a}}$ \\
\hline \multicolumn{4}{|l|}{ HR (mmHg) } \\
\hline $2 \mathrm{~h}$ & $89.5 \pm 10.2$ & $89.7 \pm 12.9$ & 0.8 \\
\hline $4 \mathrm{~h}$ & $92.2 \pm 10.2$ & $89.8 \pm 11.3$ & 0.5 \\
\hline $6 \mathrm{~h}$ & $91.8 \pm 11.5$ & $91.5 \pm 11.9$ & 0.9 \\
\hline $8 \mathrm{~h}$ & $90.8 \pm 7.3$ & $94.8 \pm 14.1$ & 0.1 \\
\hline $10 \mathrm{~h}$ & $90.0 \pm 1.0$ & $97.2 \pm 14.3$ & 0.2 \\
\hline $12 \mathrm{~h}$ & $94.0 \pm 6.5$ & $99 \pm 7.7$ & 0.3 \\
\hline
\end{tabular}

Abbreviation: MAP: mean atrial pressure, HR: heart rate.

${ }^{\text {a }}$ Significant P-value

\begin{tabular}{|c|c|c|c|c|c|c|c|c|c|c|c|c|c|}
\hline & \multicolumn{6}{|c|}{ DP Group NRS } & \multicolumn{6}{|c|}{ D Group NRS } & \multirow{2}{*}{ P-Value } \\
\hline & $0-1$ & $2-3$ & $4-5$ & $6-7$ & $8-9$ & 10 & $0-1$ & $2-3$ & $4-5$ & $6-7$ & $8-9$ & 10 & \\
\hline $2 \mathbf{h}$ & 0 & 25 & 21 & 4 & 0 & 0 & 4 & 17 & 23 & 6 & 0 & 0 & 0.100 \\
\hline $6 \mathbf{h}$ & 0 & 28 & 21 & 1 & 0 & 0 & 5 & 13 & 21 & 11 & 0 & 0 & $0.001^{\mathrm{a}}$ \\
\hline $10 \mathrm{~h}$ & 2 & 34 & 13 & 1 & 0 & 0 & 2 & 19 & 22 & 6 & 1 & 0 & $0.025^{\mathrm{a}}$ \\
\hline $14 h$ & 0 & 4 & 27 & 19 & 0 & 0 & 0 & 9 & 24 & 12 & 4 & 1 & 0.070 \\
\hline $18 \mathrm{~h}$ & 0 & 9 & 23 & 18 & 0 & 0 & 0 & 2 & 29 & 13 & 6 & 0 & $0.008^{\mathrm{a}}$ \\
\hline $22 \mathrm{~h}$ & 0 & 24 & 20 & 6 & 0 & 0 & 0 & 5 & 25 & 14 & 6 & 0 & $0.001^{\mathrm{a}}$ \\
\hline $26 h$ & 0 & 20 & 25 & 5 & 0 & 0 & 0 & 14 & 27 & 8 & 1 & 0 & 0.400 \\
\hline $30 h$ & 1 & 11 & 32 & 6 & 0 & 0 & 0 & 15 & 30 & 4 & 1 & 0 & 0.500 \\
\hline $36 h$ & 2 & 28 & 20 & 0 & 0 & 0 & 0 & 16 & 31 & 2 & 1 & 0 & $0.030^{\mathrm{a}}$ \\
\hline
\end{tabular}

Abbreviation: NRS: numerical rating pain score.

${ }^{\text {a }}$ Significant P-value

Paracetamol is one of the popular analgesics for perioperative pain management. It has a few adverse effects and no gastrointestinal side effects and is suitable for children and adults. It does not cause coagulation disorders even in patients taking anticoagulants. The drug is excreted in the urine. The analgesic mechanism of this drug is not fully understood, and there are various forms for oral, intravenous, and rectal use. Intravenous administration of paracetamol as an adjuvant to tramadol before the end of cardiac surgery, and continued for 72 hours, has reduced morphine requirement (19). This study was consistent with our study in the group that received parac- etamol and dexmedetomidine. In another study, a multimodal method was designed to compare dexamethasone, gabapentin, ibuprofen and paracetamol after cardiac surgery with the traditional method, which included morphine and paracetamol (20). The results showed the pain score decreased in the multimodal group on the day of surgery until the third day after surgery, and this was consistent with our study comparing DP group with the D group. Alhashemi et al. were studied i.v. acetaminophen compared with oral ibuprofen in the combination with morphine in the form of PCA for analgesia after cesarean section and showed that i.v. acetaminophen could be used 
as a suitable alternative to oral ibuprofen (21) Because no single method is effective, the use of a combination of techniques and analgesics can be more effective and also have fewer side effects (22-24)

\subsection{Conclusions}

This study shows that adding paracetamol to the dexmedetomidine in the PCA pump can provide better analgesia quality, reduce the need for narcotics and less dose of narcotics, and reduce the duration of intubation.

\section{Footnotes}

Authors' Contribution: Study concept and design: FMH and EH. Acquisition of data: SMM. Analysis and interpretation of data: FMH. Drafting of the manuscript: AS and RF. Critical revision of the manuscript for important intellectual content: FM. Statistical analysis: FMH.

Conflict of Interests: There was no conflict of interest.

Ethical Approval: The study was approved by the Ethics Committee of in Rajaie Heart Center (code: IR.RHC.REC.1398.042).

Funding/Support: There was no financial support for this research.

Informed Consent: Informed consent was obtained from all eligible patients preoperatively.

\section{References}

1. Mazzeffi M, KhelemskyY. Poststernotomy pain: A clinical review. J Car diothorac Vasc Anesth. 2011;25(6):1163-78. doi:10.1053/j.jvca.2011.08.001. [PubMed: 21955825].

2. Chandrakantan A, Glass PS. Multimodal therapies for postoperative nausea and vomiting, and pain. Br J Anaesth. 2011;107 Suppl 1:i27-40. doi: 10.1093/bja/aer358. [PubMed: 22156268]

3. Fayaz MK, Abel RJ, Pugh SC, Hall JE, Djaiani G, Mecklenburgh JS Opioid-sparing effects of diclofenac and paracetamol lead to improved outcomes after cardiac surgery. J Cardiothorac Vasc Anesth. 2004;18(6):742-7. doi:10.1053/j.jvca.2004.08.012. [PubMed:15650984].

4. Rupniewska-Ladyko A, Malec-Milewska M. A high dose of fentanyl may accelerate the onset of acute postoperative pain. Anesth Pain Med. 2019;9(5). e94498. doi: 10.5812/aapm.94498. [PubMed: 31903331]. [PubMed Central: PMC6935250].

5. Malik KM, Imani F, Beckerly R, Chovatiya R. Risk of opioid use disorder from exposure to opioids in the perioperative period: A systematic review. Anesth. Pain. Med.2020;10(1). doi:10.5812/aapm.101339.

6. Imani F, Zaman B, De Negri P. Postoperative pain management: Role of dexmedetomidine as an adjuvant.Anesth.Pain.Med.2021;10(6). doi: 10.5812/aapm.112176.

7. Asri S, Hosseinzadeh H, Eydi M, Marahem M, Dehghani A, Soleimanpour $\mathrm{H}$. Effect of dexmedetomidine combined with inhalation of isoflurane on oxygenation following one-lung ventilation in thoracic surgery. Anesth Pain Med. 2020;10(1). e95287. doi: 10.5812/aapm.95287. [PubMed: 32309196]. [PubMed Central: PMC7145426].

8. Amiri HR, Ohadian Moghadam S, Momeni SA, Amini M. Preemptive analgesia with a second dose of pregabalin, acetaminophen, naproxen, and dextromethorphan: A comparative clinical trial in major surgeries. Anesth Pain Med. 2020;10(5). e100718. doi: 10.5812/aapm.100718. [PubMed: 34150558]. [PubMed Central: PMC8207851]
9. Imani F, Faiz HR, Sedaghat M, Hajiashrafi M. Effects of adding ketamine to fentanyl plus acetaminophen on postoperative pain by patient controlled analgesia in abdominal surgery. Anesth Pain Med. 2014;4(1). e12162. doi: 10.5812/aapm.12162. [PubMed: 24660145]. [PubMed Central: PMC3961015].

10. Imani F, Rahimzadeh P, Faiz HR, Nowruzina S, Shakeri A, Ghahremani M. Comparison of the post-caesarean analgesic effect of adding dexmedetomidine to paracetamol and ketorolac: A randomized clinical trial. Anesth Pain Med. 2018;8(5). e85311. doi: 10.5812/aapm.85311. [PubMed: 30538943]. [PubMed Central: PMC6252045].

11. Liu SS, Wu CL. Effect of postoperative analgesia on major postoperative complications: a systematic update of the evidence. Anesth Analg. 2007;104(3):689-702. doi: 10.1213/01.ane.0000255040.71600.41. [PubMed: 17312231].

12. Beattie WS, Badner NH, Choi PT. Meta-analysis demonstrates statistically significant reduction in postoperative myocardial infarction with the use of thoracic epidural analgesia. Anesth Analg. 2003;97(3):919-20. doi: 10.1213/01.ane.0000074660.05733.10. [PubMed: 12933434].

13. Kehlet H, Jensen TS, Woolf CJ. Persistent postsurgical pain: risk factors and prevention. Lancet. 2006;367(9522):1618-25. doi: 10.1016/s0140 6736(06)68700-x.

14. Moon MH, Kang JK, Kim HW, Jo KH, Choi SH, Song H. Pain after median sternotomy: collateral damage or mitigatable byproduct? Thorac Cardiovasc Surg. 2013;61(3):194-201. doi: 10.1055/s-0032-1311540. [PubMed: 23132359].

15. Janatmakan F, Nassajian N, Jarirahmadi S, Tabatabaee K, Zafari M Comparison of the effect of dexmedetomidine and remifentanil on pain control after spinal surgery: A double-blind, randomized clinical trial. Anesth. Pain. Med.2021;11(2). doi: 10.5812/aapm.111533.

16. Oriby ME, Elrashidy A. Comparative effects of total intravenous anesthesia with propofol and remifentanil versus inhalational sevoflurane with dexmedetomidine on emergence delirium in children undergoing strabismus surgery. Anesth. Pain. Med. 2020;11(1). doi: 10.5812/aapm.109048.

17. Ibrahim IM, Hassan R, Mostafa RH, Ibrahim MA. Efficacy of dexmedetomidine infusion without loading dose on hemodynamic variables and recovery time during craniotomy: A randomized double-blinded controlled study. Anesth. Pain. Med. 2021;11(2). doi: 10.5812/aapm.113410.

18. El Mourad MB, Elghamry MR, Mansour RF, Afandy ME. Comparison of intravenous dexmedetomidine-propofol versus ketofol for sedation during awake fiberoptic intubation: A prospective, randomized study. Anesth Pain Med. 2019;9(1). e86442. doi: 10.5812/aapm.86442. [PubMed: 30881913]. [PubMed Central: PMC6412910].

19. Cattabriga I, Pacini D, Lamazza G, Talarico F, Di Bartolomeo R, Grillone $\mathrm{G}$, et al. Intravenous paracetamol as adjunctive treatment for postoperative pain after cardiac surgery: a double blind randomized controlled trial. Eur J Cardiothorac Surg. 2007;32(3):527-31. doi 10.1016/j.ejcts.2007.05.017. [PubMed:17643995].

20. Rafiq S, Steinbruchel DA, Wanscher MJ, Andersen LW, Navne A Lilleoer NB, et al. Multimodal analgesia versus traditional opiate based analgesia after cardiac surgery, a randomized controlled trial. J Cardiothorac Surg. 2014;9:52. doi: 10.1186/1749-8090-9-52. [PubMed: 24650125]. [PubMed Central: PMC3975463].

21. Alhashemi JA, Alotaibi QA, Mashaat MS, Kaid TM, Mujallid RH, Kaki AM. Intravenous acetaminophen vs oral ibuprofen in combination with morphine PCIA after Cesarean delivery. Can J Anaesth. 2006;53(12):1200-6. doi:10.1007/BF03021581. [PubMed:17142654].

22. Myles PS, Daly DJ, Djaiani G, Lee A, Cheng DC. A systematic review of the safety and effectiveness of fast-track cardiac anesthesia. Anesthesiology. 2003;99(4):982-7. doi: 10.1097/00000542-200310000-00035. [PubMed: 14508335].

23. Alavi SM, Baharestani B, Fariborz FB, Bakhshandeh $\mathrm{H}$, Babaee $T$, Sadeghpur A, et al. Intraoperative magnesium sulfate can reduce narcotic requirement after coronary bypass surgery. IHJ. 2011;12(1):6-11.

24. Alavi SM, Kish RF, Farsad F, Imani F, Sheikhvatan M. Intravenous sufentanil and morphine for post-cardiac surgery pain relief using patientcontrolled analgesia (PCA) device: A randomized double-blind clinical trial. Pak. J. Med. Sci. Q. 2010;26(1):137-41. 